\title{
L'intelligence collective : un enjeu politique
}

Collective intelligence: A political issue

\section{Gérald Bronner}

\section{OpenEdition}

\section{Journals}

Édition électronique

URL : https://journals.openedition.org/ress/4454

DOI : $10.4000 /$ ress. 4454

ISSN : 1663-4446

\section{Éditeur}

Librairie Droz

\section{Édition imprimée}

Date de publication : 14 décembre 2018

Pagination : 161-182

ISSN : 0048-8046

\section{Référence électronique}

Gérald Bronner, «L'intelligence collective : un enjeu politique », Revue européenne des sciences sociales [En ligne], 56-2 | 2018, mis en ligne le 14 décembre 2021, consulté le 06 janvier 2022. URL : http:// journals.openedition.org/ress/4454; DOI : https://doi.org/10.4000/ress.4454 


\title{
L'INTELLIGENCE COLLECTIVE: UN ENJEU POLITIQUE
}

\author{
GÉRALD BRONNER \\ Université Paris-Diderot, LIED \\ gerald.bronner@univ-paris-diderot.fr
}

\begin{abstract}
Résumé. De nombreux penseurs et acteurs du politique considèrent que les formes collaboratives de la démocratie offrent des solutions pour rénover nos systèmes politiques. Sans contester ni approuver cette idée, cet article constate que les enjeux idéologiques qu'elle implique sont de nature à masquer certains des problèmes techniques qu'elle soulève. La question la plus cruciale étant sans doute celle-ci: à quelles conditions la décision collective aboutit-elle à des formes de conclusions sages ou au contraire à des erreurs sévères? Le texte, après avoir rappeler un certain nombre de résultats expérimentaux, s'adosse à une étude originale impliquant 216 sujets volontaires dans des focus groups pour proposer une contribution à ce problème en distinguant notamment deux types de situations cognitives bien distinctes: les problèmes «galtoniens» et les problèmes de biais.
\end{abstract}

Mots-clés: biais cognitifs, consensus, intelligence collective, irrationalité collective, polarisation, sagesse des foules.

\begin{abstract}
Many thinkers and policymakers believe that collaborative forms of democracy offer solutions to renew our political systems. Without contesting or endorsing this idea, this article notes that the ideological assumptions on which such an idea rests tend to mask some of the technical problems it raises. The most crucial issue is perhaps the following: under what conditions does collective decision-making lead to wise conclusions or, on the contrary, to severe errors? The text, after recalling a certain number of experimental results, draws on an original study involving 216 volunteers in focus groups and addresses this problem by distinguishing between two distinct types of cognitive situations: "Galtonian" problems and problems of bias.
\end{abstract}

Keywords: cognitive bias, collective intelligence, collective irrationality, consensus, polarization, wisdom of crowds. 
INTRODUCTION

Les compétences collectives ne sauraient être considérées toujours comme la sommation des compétences individuelles. Ainsi, s'il est bien vrai que dix hommes peuvent lever une masse dix fois plus importante qu'un seul, il serait risible de considérer que les dix mêmes courent dix fois plus vite qu'un seul. Le partage entre les situations de mutualisation de l'effort dans les activités physiques collectives n'est pas difficile à opérer. Les choses sont plus complexes lorsque l'on tente d'éclairer les rapports qu'entretiennent cognition individuelle et cognition collective. Raisonne-t-on mieux tout seul ou à plusieurs ? Est-on plus rapide à résoudre un problème si l'on mutualise ses efforts intellectuels ou, au contraire, la dynamique mentale des groupes constitue-t-elle un obstacle à l'expression du raisonnable? C'est une question presque aussi ancienne que l'histoire des idées mais qui revêt une grande actualité. Que ce soit par le processus choisi lors du débat national sur la transition énergétique en France lancé en 2012 ou par les tâtonnements observés lors du mouvement « Nuit debout», le thème de la délibération est au cœur de la plupart des réflexions concernant le renouveau démocratique.

Cette idée de la participation élargie des citoyens aux décisions politiques ne va pas de soi. L'hypothèse qui a paru s'imposer d'abord dans l'histoire des idées est que les foules sont irrationnelles et que la rationalité individuelle s'y perd comme une goutte dans un lac. On peut trouver cette thèse aussi bien chez Platon, qui considère par exemple que le peuple ne peut, par essence, être philosophe', ou même chez des auteurs qui ont consacré des livres entiers à démontrer la stupidité des foules, comme Gustave Le Bon (I895 [2003]) ou Charles Mackay (1845 [1995]).

$A$ contrario, on a vu se développer dans la deuxième partie du $\mathrm{xx}^{\mathrm{e}}$ siècle, à partir des années 1960, des revendications concernant la démocratie participative puis délibérative qui partait de postulat inverse (voir Girard et Legoff, 2010). Des théoriciens comme Carole Pateman (1970) et Benjamin Barber (1984), notamment, font de l'implication de chacun dans les affaires publiques, 
la condition de toute liberté politique authentique. Cette conception participative de la démocratie, où chacun a le droit de contribuer à la décision, d'être consulté, a notamment pris corps aux États-Unis dans un contexte de vagues protestataires (mouvement des droits civiques, lutte contre la guerre du Vietnam, etc.). Ces propositions de philosophie politique trouvent bientôt un débouché avec toutes sortes de dispositifs visant à permettre aux citoyens d'être partie prenante de certaines décisions collectives qui peuvent relever tout aussi bien de l'aménagement urbain, de questions de gestion budgétaire ou encore d'évaluation d'innovation technologique. On les nomme : commissions de quartier, publiforums, conférences citoyenne, auditions et enquêtes publiques, focus groups, jurys citoyens, procédures de consultation, consultations riveraines, budgets participatifs, forums, conférences de consensus, etc.

L'idée est double, d'une part rendre les processus de décision publique plus transparents et, d'autre part, permettre aux hommes politiques, jugés trop éloignés des citoyens, de pouvoir écouter ce qu'ils ont à leur dire entre les élections. On les a vus apparaître aux États-Unis dans les années I960 et 1970, et en France dans les années 1980. Dans notre pays, elle prend, par exemple, la forme de l'enquête publique et notamment de sa tentative de démocratisation par la loi Bouchardeau du 12 juillet 1983: il s'agit alors, non seulement d'informer le public, mais encore de recueillir ses suggestions et ses contre-propositions.

Le thème de la participation citoyenne remet sur le devant de la scène une question démocratique fondamentale, celle qui oppose l'opinion publique et l'intérêt général. En effet, garantir la participation de tous à une décision et garantir la sagesse de cette décision constituent deux opérations distinctes. Il n’est pas illégitime en démocratie de considérer la possibilité de la participation de tous à la décision politique (le cas ultime étant le référendum), mais comment être certain que cette collaboration que l'on approuve du point de vue des principes ne sera pas catastrophique du point de vue de ses conséquences?

Pour répondre à cette évidente objection, la démocratie participative s'est souvent muée en démocratie délibérative. Le terme de démocratie délibérative est né d’une expression proposée par Joseph Bessette, en I980, pour qualifier la pratique des membres du Congrès américain. L’idée des défenseurs de la démocratie 
Tiré à part adressé à Gérald Bronner

délibérative est que la sagesse n'émerge pas spontanément d'une assemblée qui décide, la consultation ne serait donc pas suffisante. Cette sagesse, en revanche, sera au rendez-vous si l'on permet à cette assemblée de suivre une formation et de délibérer librement sur le sujet sur lequel elle doit prendre position. Pour cette théorie démocratique «la délibération publique et libre entre citoyens égaux constitue le fondement de la légitimité politique» (Girard et Legoff, p. I I).

Les spécialistes de la question ont vigoureusement distingué les notions de démocraties participatives et délibératives puisqu’elles ne renvoient pas conceptuellement aux mêmes processus de décisions (la seconde impliquant nécessairement la première mais pas l'inverse). Dans les faits, notamment en France, il semble qu'on utilise plus fréquemment la première notion que la seconde, y compris dans des processus impliquant, en fait, la délibération. Il semble donc qu’en dehors des différences conceptuelles impliquées, le marqueur linguistique soit dominant comme le montre les deux graphiques suivants obtenus grâce à logiciel Google books Ngram Viewer (qui permet entre autres d'évaluer la fréquence de l'utilisation d'un terme dans les I5 millions d'ouvrages numérisés par Google).

Graphiques I Fréquence des termes «deliberative democracy» et "participative democracy»

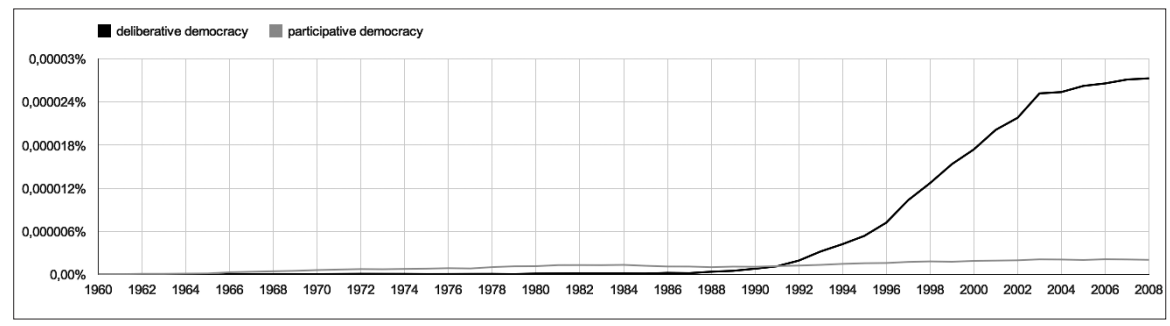

Graphique 2. Fréquence des termes «démocratie délibérative» et « démocracie participative»

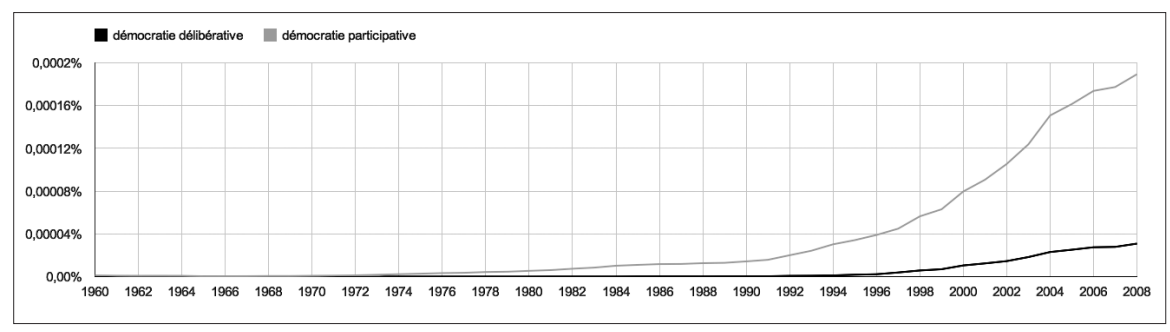


On voit donc que l'utilisation des termes en anglais ou en français n'aboutit pas du tout aux mêmes résultats, mais dans les deux cas, on voit nettement que le surgissement de ces concepts dans la littérature est notable à partir du début des années 1990 .

On attribue alors beaucoup de vertu à la délibération collective : elle encourage les individus à améliorer leur raisonnement et à avancer leurs meilleurs arguments pour convaincre les autres. De la même façon, son caractère public est une dissuasion pour chacun d'avancer des idées indécentes (Elster, 1988). Elle permet encore une meilleure circulation de l'information et conduit, de ce fait, à faire reculer les idées douteuses, les préjugés, les erreurs (Landemore, 2010; Morel, 20I2). Bref, nous explique-t-on, nous pouvons faire confiance à la délibération publique pour produire des décisions informées et conformes à l'intérêt général. Des expérimentations, parfois de grandes ampleurs sont réalisées, comme celles de James Fishkin (2009) concepteur et défenseur des «sondages délibératifs». Il s'agit pour lui de réunir des centaines de personnes à qui l'on donne une formation sur tel ou tel sujet et ensuite, après délibération, de consulter leur point de vue collectif. En janvier 2003 a eu lieu, à Philadelphie, une «votation de la Convention délibérative sur les Questions nationales» inspirée justement des travaux de Fishkin et, aujourd'hui, des centaines de villes se sont livrées à l'expérience un peu partout dans le monde. L'idée est toujours la même : le débat politique ne doit pas être confisqué par les experts et les élites politiques.

Le pays où se sont déroulées les premières expérimentations délibératives est le Danemark où sont organisées ce que l'on nomme des «conférences de consensus ». En 1985, en effet, le bureau danois de la technologie propose de mettre au cour de ces conférences un panel de citoyens profanes. Entre 1987 et 2002 ce ne sont pas moins de 22 conférences de consensus qui sont organisées sur des thèmes aussi divers que «la surveillance électronique», «les aliments transgéniques» «agriculture et écologie», etc. Beaucoup d’autres pays ont tenté l'expérience comme l'Allemagne avec sa conférence des citoyens en 200 sur les tests génétiques, ou l’Australie en 1999 sur les technologies géniques dans la chaîne alimentaire. 
Tiré à part adressé à Gérald Bronner

Les notions de démocraties délibératives et participatives ont donc nombre d'implications pratiques mais ce n'est pas toujours cet aspect qui a primé dans l'histoire des idées. En effet, la méfiance à l'égard du pouvoir est consubstantielle à l'histoire de la démocratie (Rosenvallon, 2006). Cependant, il semble que cette méfiance prenne, à certaines périodes, une dimension inquiétante. Plusieurs enquêtes montrent que ce sentiment de méfiance touche aussi bien le pouvoir politique, médiatique, que l'expertise scientifique (Bronner, 2003). Une des solutions envisagées pour faire reculer ce sentiment est de développer les instances collaboratives entre ceux qui paraissent détenir le pouvoir, même lorsqu'il n'est que symbolique, et ceux qui constituent, ce que l'on désigne d’une métaphore pas toujours très claire, le peuple. Que ce soit en se servant des nouvelles possibilités offertes par Internet ou des processus de démocratie participative ou délibérative, beaucoup d'auteurs défendent l'idée qu'il serait urgent de «démocratiser la démocratie» (Callon, Lascoumes, et Barthe, 200I). Enfin, écrivent certains (voir, notamment, Leadbeater et Miller, 2004 ou Flichy, 20Io), la démocratie va arriver à son âge de maturité, elle qui souffrait précédemment d'une forme d'infantilisme. Ainsi Dominique Cardon (2010) indique clairement que pour lui «Internet est un instrument de lutte contre l'infantilisation des citoyens dans un régime qui est censé leur confier le pouvoir. En ce sens le web incarne l'avenir de la démocratie. »

Cet espoir est parfaitement résumé par Benjamin Loveluck (2008) : «une formidable promesse d'égalité [...] et surtout l'utopie d'une parole libre, sans instance de censure, voire un idéal de démocratie participative fondée sur la délibération permanente $[\ldots]$ la réalisation en cours d'une utopie politique à part entière : celle de la démocratie dans sa forme la plus pure ».

Ces auteurs ont souvent un rapport inconditionnel à cette idée de sorte qu'elle peut prendre les atours de ce qui est une nouvelle forme de passion politique. Je consacrerai cet article à tenter de désincarcérer ce problème important de sa gangue idéologique en m’attachant à le saisir dans sa portée épistémique plutôt qu’axiologique. 


\section{LE CEUR DE LA QUESTION DE L'INTELLIGENCE COLLECTIVE}

On pourrait consacrer beaucoup de pages à énoncer les bienfaits de l'intelligence collective comme ses méfaits (Bronner, 20I4). Ainsi, on sait que les efforts individuels de mutualisation de l'information aboutissent concrètement à l'amélioration du savoir collectif. Par exemple la mobilisation d'un grand nombre d'observateurs, des phénomènes à occurrence rare, notamment dans le domaine médical, a permis de révéler l'existence de certains symptômes atypiques. Les foules paraissent encore extraordinairement compétentes lorsqu'une tâche demande des capacités exploratoires. Le jeu Foldit, par exemple, propose aux internautes de tenter librement des combinaisons moléculaires pour mieux cerner la façon dont les protéines peuvent se déployer dans l'espace : il s'agit de déplacer des portions ici, d'en ajouter là ou même de détruire des liaisons. Ce jeu collectif de construction a permis la publication de trois articles dont l'un dans la très prestigieuse revue Nature. Symétriquement, la mutualisation des efforts intellectuels paraît parfois contre-productive. C'est le cas, par exemple, lorsqu'on observe un effet dit de «cascade». Ces phénomènes surgissent dans un groupe qui succombe à une forme de conformisme cognitif: la majorité étant incertaine, elle aura tendance à s'indexer sur une minorité ayant déjà un point de vue sur tel ou tel sujet. Il se trouve dans tout groupe des individus bénéficiant d'un prestige, d'une élocution, d'un niveau social et culturel, etc., leur permettant de se différencier des autres et de prendre la parole en premier (Mendelberg, 2002). Il existe des effets de «polarisation» qui se manifestent lorsqu'un groupe ayant délibéré adopte des positions plus radicales que la moyenne des positions individuelles avant la discussion. C’est parce que ce phénomène est extrêmement fréquent (y compris parmi des groupes n'ayant pas de disposition apparente à la radicalité) qu'il constitue un argument souvent retenu pour tempérer l'enthousiasme des partisans de la sagesse des foules. On a pu l'observer expérimentalement sur des sujets tels que l'évaluation des politiques d’aides sociales aux États-Unis, le féminisme, le préjugés racistes, etc. Comme l'explique Cass Sunstein (2006), certains individus, dans la délibération, vont avoir tendance à s'engager dans une concurrence déclarative, affichant des positions censées attester leur sincérité et entraînant une partie du groupe dans des formes de surenchères. Par ailleurs, le processus d’argumentation confrontera 
Tiré à part adressé à Gérald Bronner

chacun à un «fond» d'arguments propre à chaque groupe et exposera les individus à une forme de cohérence qui réclamera souvent la radicalité. Comme le font remarquer les politologues S. Brock Blomberg et JosephE. Harrington (2000), les individus les plus radicaux (que sont souvent les militants) auront probablement des arguments plus percutants (parce qu’ils auront déjà été éprouvés) et le moins de difficulté à prendre la parole en public pour les exprimer.

On sait enfin que les délibérations locales peuvent fractionner l'intérêt général en intérêts particuliers, c'est notamment le cas dans les célèbres effets NIMBY' : ainsi l'implantation d'un centre de retraitement des déchets ou d'un champ d'éolienne ne passe pas aisément le cap de la démocratie délibérative. Ces résultats contrastés étant connus et documentés, je m’attacherai à une question plus essentielle, non parce qu'elle est plus importante que toutes les autres, mais parce qu'elle définit l'essence du problème.

Cette questions essentielle est exprimable de cette façon : à quelle condition un groupe qui décide (par agrégation de choix individuels ou par délibération) aboutit-il à des décisions optimales, sub-optimales ou même contre-productives ? La réponse à cette question ne paraît pas mystérieuse aux défenseurs inconditionnels de la sagesse des foules, ainsi James Surowiecki (2008 [2004], p. 24), l'un des avocats le plus connus de cette idée avance-t-il : «Que l'on réunisse un groupe d'individus suffisamment important et diversifié et qu'on lui demande de "prendre des décisions en matière d’intérêt général", les décisions de ce groupe seront, au fil du temps, "intellectuellement supérieures à celles de l’individu isolé”, aussi intelligent ou bien informé soit-il.»

Les résultats de l'évaluation de ce postulat sont essentiels pour apprécier les espoirs que suscite l'idée de travail collaboratif. Pour ce faire, il faut distinguer fermement deux processus idealtypiques de décision collective. Le premier résulte de l'agrégation de préférences individuelles tandis que le second procède d'une délibération qui peut aboutir à une unanimité, un consensus ou à un point de

2 NIMBY est l'acronyme de «not in my backyard», il désigne habituellement l'opposition à un projet par des riverains qui craignent les nuisance de proximité. À titre d'exemple, le village de Sousey-sur-Brionne a-t-il fait savoir, lorsqu'on la consulté en 2015, qu'il ne tenait pas à ce que des éoliennes soient implantées sur son territoire (à $80 \%$ des suffrages exprimés). 
vue majoritaire. Sans doute la seconde formule paraît-elle intuitivement plus démocratique puisqu'elle mise sur les vertus supposées performantes du débat. Pourtant, c'est bien par un exemple du premier type de sagesse des foules que Surowiecki inaugure son livre : la célèbre «expérience » de Galton. Francis Galton est un anthropologue et statisticien britannique, promoteur de l'eugénisme, il fit en 1906 une constatation qui le surprit. Assistant dans un marché de bétail à un concours mettant au défi les participants d'évaluer les poids exact d'un bœuf, il recueillit les 787 paris individuels. Le calcul de la médiane de ces paris lui permet d'observer que «la foule» aboutissait à une réponse extrêmement performante (I197 livres alors que le poids réel du bœuf était de I198).

Kate Gordon (1924) a mené des expériences similaires au début du $\mathrm{xx}^{\mathrm{e}}$ siècle, elle demanda à des centaines d'étudiants d'évaluer individuellement le poids d'un certain nombre d'objets présentés devant eux en les classant par ordre croissant: du plus léger au plus lourd. L'exercice pouvait paraître saugrenu: personne dans l'assemblée n’avait de compétence particulière pour le résoudre, hormis l'expérience ordinaire que chacun peut avoir de la masse des objets. Certains se montrèrent plus habiles que d'autres à cet exercice. Ce que ses étudiants ignoraient, c'est qu'ils participaient à une étude visant non à évaluer leur compétence personnelle, mais plutôt leur compétence collective. En d'autres termes, ces étudiants allaient-ils être plus compétents collectivement ou individuellement pour résoudre le problème? Les résultats de l'expérimentation montrent que l'évaluation du groupe fut exacte dans $94 \%$ des cas. Cette moyenne fut plus élevée que toutes les évaluations individuelles à l'exception de cinq d'entre elles. On a souvent reproduit ce type d'expériences, la plus classique étant de faire évaluer le nombre de bonbons contenus dans un bocal. C'est notamment le cas pour une étude menée par Jens Krause, Graeme D. Ruxton et Stefan Krause (2010). Ils ont demandé à 2057 individus d'évaluer le nombre de billes dans une jarre en contenant 562. Les individus ne se consultaient pas les uns les autres, comme dans l'observation de Galton. Les indices de centralité révèlent que l'agrégation des évaluations collectives est très performante. Ainsi, la moyenne des réponses est de 554 et la médiane de 516. 
Tiré à part adressé à Gérald Bronner

Immanquablement, on observe que l'évaluation du groupe est supérieure à la grande majorité des appréciations individuelles et que si l'on doit se fier à une décision, mieux vaut en toute probabilité choisir un indice de centralité agrégeant les points de vue individuels qu'un point de vue exprimé en particulier. Ces résultats n'ont rien de surprenant en réalité, il suffit de considérer qu'en cas de doute (ici le classement exact des objets selon leur poids, le nombre de billes contenues dans le bocal) tous se trompent, parce qu'il est très difficile de donner la bonne réponse, mais que leur erreur est équirépartie. En d'autres termes, les estimations par excès des uns compensent les estimations par défaut des autres. C'est pourquoi les indices de centralité constituent parfois une excellente aide à la décision. Mais l'enthousiasme des défenseurs de la sagesse des foules leur a parfois fait perdre de vue qu'il s'agit là d'une structure d’agrégation de choix individuels bien spécifiques. À ce niveau de notre discussion, il nous faut évaluer si ce mécanisme d'équirépartition de l'erreur fonctionne de la même manière lorsqu'il ne relève pas de l'agrégation aveugle des réponses individuelles mais d’une délibération?

\section{L'INTELLIGENCE COLLECTIVE À L'ÉPREUVE DE L'ÉQUIRÉPARTITION DE L'ERREUR}

Pour tester cette idée, nous avons réalisé 54 focus groups ${ }^{3}$ impliquant 216 individus entre janvier 2015 et janvier 2016. Chaque groupe était composé de quatre personnes choisies en fonction de leur sexe et de leur âge afin de former des groupes variés. Le critère de recrutement le plus important est qu'ils ne se connaissaient pas afin d'éviter autant que possible les effets d’autorité relatifs à leurs interactions passées. Les individus n’étaient pas informés du sujet de l'expérience, mais ils avaient la garantie de la préservation de leur anonymat et savaient que leurs propos seraient enregistrés. Les focus groups n’ont pas pu être tous réalisés dans le même lieu (certains ont été conduit dans une salle à l’uni-

3 En fait 27 et 27 car deux problèmes différents furent posés: ils nous a paru préférable méthodologiquement de ne pas poser ces deux problèmes aux mêmes groupes. Les uns étaient confrontés à un problème «galtonien», les autres à un problème de biais cognitif, comme nous le verrons plus bas. 
versité Paris 7 dévolue à cet usage, d’autres chez des enquêteurs recrutés à cette occasion). D’une façon générale, ces expérimentations n’ont pas été réalisées dans des lieux publics. Elles commençaient toujours par un entretien individuel permettant de recueillir des données factuelles (âge, profession etc.) en confidentialité pour limiter l'influence possible des représentations sociales. Une fois les entretiens individuels réalisés, chacun était réuni autour d'une table avec un expérimentateur dont le but était seulement de favoriser la prise de parole par des relances aussi neutres que possibles, telles qu'elles peuvent se pratiquer dans les entretiens non-directifs. Il est par ailleurs indiqué aux participants, qu'ils ne pourront utiliser ni calculatrice, ni feuille de papier, téléphone ou ordinateur pour poser le problème. Le problème leur est alors présenté, il s’agit d'évaluer le nombre de lentilles dans un flacon (voir la photo ci-dessous) qui est posé au milieu de la table.

Les 562 lentilles de l'expérimentation $\mathrm{n}^{\circ}$ I

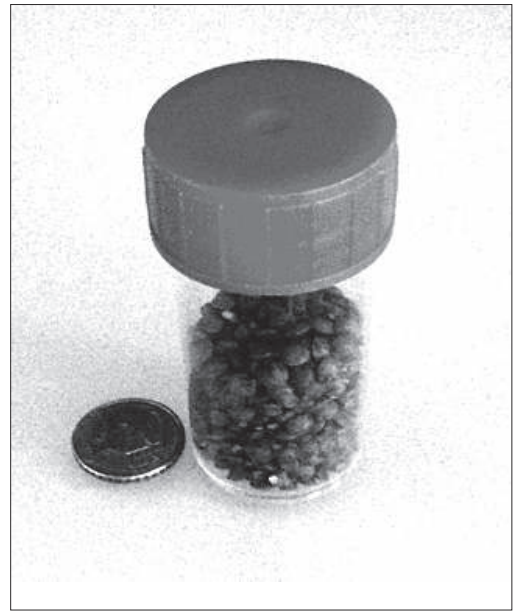

Il leur est précisé qu'ils n'ont pas le droit de toucher ce flacon pour le soupeser afin de décompter les lentilles. L’expérimentation peut commencer soutenue par l'expérimentateur qui demande à chacun de se prononcer pour lancer les échanges. Lorsque les échanges s'essoufflent, le temps du chronomètre est noté une première fois sans être interrompu, puis il est demandé au 


\section{Tiré à part adressé à Gérald Bronner}

groupe de former un consensus, de s'entendre sur une seule réponse. Une fois confirmée par l'ensemble du groupe, le dictaphone peut être arrêté.

L'expérience est ensuite strictement retranscrite. On relève les différentes évaluations, la valeur de consensus, le temps pris pour y arriver, le nombre de mots prononcés par chacun. À titre d'exemple, on obtient ce type de représentation graphique.

Figure I. Exemple de résultats pour un groupe

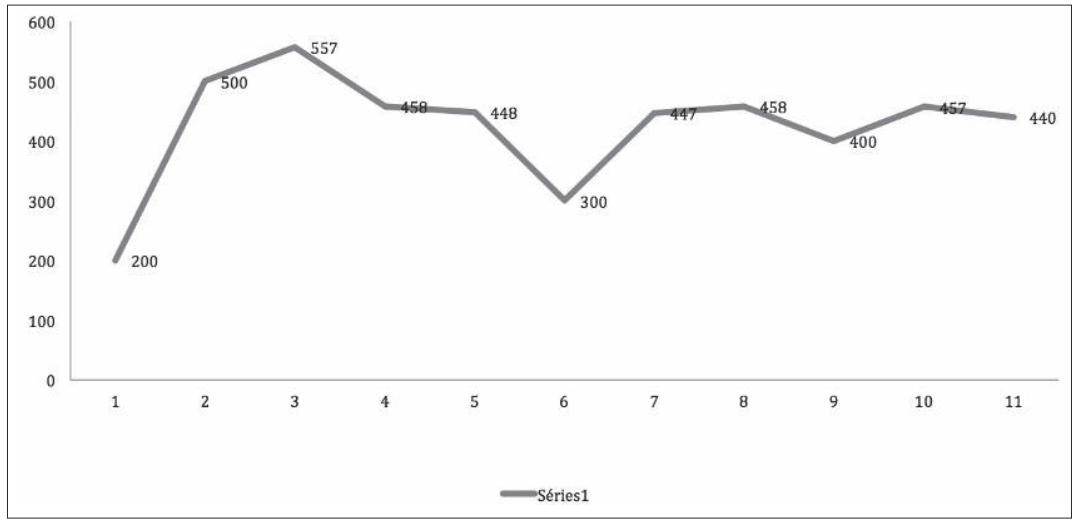

Note: Valeur du consensus: 444 - Nombre de mots échangés: 631

Si l'on compare les résultats obtenus dans cette expérimentation et ceux obtenus par exemple dans l'expérience de Krause, Ruxton et Krause (où le nombre d'éléments contenus dans un bocal était le même: 562) on observe que la performance de ce qu'on peut appeler l'intelligence collective n'est pas du tout la même. En effet, si l'on retient l'indice de centralité le plus performant (ici la moyenne) dans le processus d’agrégation des évaluations individuelles, le taux d'erreur est de I,5\% alors que dans le cas de la solution trouvée par délibération ${ }^{4}$ il est de $34 \%$. Il n'y a sans doute pas lieu de s'étonner de ce résultat dans la mesure où l'effet de sagesse des foules dans des expériences «galtoniennes» est obtenu grâce au mécanisme d'équirépartition de l'erreur. Cet effet est contrarié dans la délibération par certains des effets que

4 On retenait la valeur du consensus lorsqu'il pouvait être établi, c'est-à-dire la majorité des cas et la moyenne des points de vue individuels lorsque ce n'était pas possible. 
nous avons mentionnés plus haut (polarisation ou ancrage), mais ils ressortent aussi sans doute des effets de conformisme cognitif qui ont été bien documentées par la psychologie sociale. Ces effets sont connus notamment par les expériences de Asch (1946 et 1955) sur le conformisme cognitif, mais ont été confirmés internationalement à plusieurs reprises (Sunstein, 2003). Ils avaient d’ailleurs été précédés par une série d’expériences moins connues menées par Muzafer Shérif publiées en 1937 et convergeant vers le même type de résultats. Le psychologue turc plaçait les sujets de son expérience dans une pièce sombre en leur demandant d'observer un point lumineux immobile. Comptant sur l'illusion due à l'effet autocynétique, il demandait à chacun d'évaluer la distance parcourue par le point lumineux. Il observait alors un effet de dispersion des réponses comme dans la tâche de Galton, mais cet effet disparaissait au profit d'un effet de conformité dès lors qu'il proposait aux sujets de délibérer en petits groupes pour évaluer collectivement cette distance illusoirement parcourue. Plus récemment, Ali Mahmoodi et al. (2015) ont montré l'existence d'un «prisme de l'équité » dans les délibérations collectives qui aboutit à affaiblir la considération des faits par rapport à celle des valeurs consensuelles.

\section{L'INTELLIGENCE COLLECTIVE À L'ÉPREUVE DES BIAIS COGNITIFS}

L’expérience de Shérif est intéressante, quoiqu’ancienne, car il y a bien une bonne réponse à la question posée (qui est o : la distance parcourue) mais l'on observe une convergence collective vers l'erreur qui est la conséquence d'une illusion contrairement à la situation de Galton où l'on attend une équirépartition de l'erreur favorable à l'expression de «la sagesse des foules». Nous avons vu que cette équirépartition pouvait être perturbée par la délibération collective plutôt qu’améliorée. Mais qu’en est-il d'une structure de situation bien différente où, comme dans l'expérience de Shérif, on peut s'attendre à une convergence statistique des points de vue individuels vers l'erreur? Ces situations sont bien documentées notamment lorsqu'elles relèvent non d'illusion d'optique 
Tiré à part adressé à Gérald Bronner

mais de l'existence de ce que la littérature a nommé des «biais cognitifs »5. Certains énoncés ou structures de situation nous conduisent à répondre en toute probabilité de façon fautive. C'est parce qu'ils ont voulu comparer l'efficacité de l’agrégation des réponses individuelles dans un problème «galtonien» et dans un problème «à biais cognitif» que Krause, Ruxton et Krause (20I0) ont confronté 1953 individus, symétriquement au problème de l'évaluation du nombre de billes dans une jarre, à une question relevant d'une évaluation probabiliste lesté par un biais cognitif. Il s'agissait d'estimer le nombre de fois à la suite où, dans un jeu de pile ou face, il fallait obtenir «face» pour approcher la probabilité de chances de gagner à la loterie allemande (I chance sur I4 millions). La bonne réponse était qu'il faut obtenir 24 «faces » de suite pour approcher les faibles chances de gagner à la loterie. Or, cette fois, l’agrégation des points de vue individuels a aboutit à des indices de centralité peu performants. Ainsi, la moyenne des réponses fut de 498 et la médiane de ıoo.

De la même façon, il est permis de se demander quel est l'impact de la délibération sur la performance de la réponse collective? C'est pour répondre à cette question que nous avons intégré dans nos expérimentations un problème relevant d'un biais cognitiff. Selon le même protocole que celui décrit précédemment, nous avons confrontés I08 personnes dans 27 focus groups au problème suivant:

Supposons que l'on vous propose de jouer au jeu suivant : voici deux cartes, l'une est rouge sur le recto et le verso, l'autre est rouge sur le recto et blanche sur le verso. Je tire au hasard une de ces deux cartes et je la pose sur une table: la face apparente est rouge. À votre avis,

- ce sera plus probablement la carte blanche-rouge;

- ce sera plus probablement la carte rouge-rouge;

- l'une ou l'autre éventualité sont aussi probables?

5 Pour une synthèse collective, voir Tversky, Kahneman et Slovic, 1984; et plus récemment Kahneman, 2012. Pour une approche critique de l'interprétation de ces résultats, voir Bronner, 2007.

6 Notre intention était initialement de reprendre le problème de Krause, Ruxton et Krause afin de comparer leurs résultats aux nôtres, comme nous l'avons fait dans le cas des expériences «galtoniennes». II se trouve que l'énoncé proposé par eux n'était tout simplement pas compris dans les focus groups exploratoires que nous avons menés. Nous avons donc préféré un autre énoncé plus simple à propos duquel nous avions aussi des résultats par agrégation importants qui nous permettaient de faire une comparaison. 
La bonne réponse à ce problème est que la carte rouge-rouge, dans les conditions définies, sera probablement [66,66\% de chances] la carte tirée au hasard.

Du mois de décembre 2002 au mois de mai $2003^{7}$, nous avons conduit une enquête quantitative par stratification. L'échantillon fut stratifié selon le niveau d'étude avec une première moitié étant constituée d'individus n'ayant pas le baccalauréat (775 personnes), et une seconde d'individus ayant au moins un bac plus trois (784 personnes). On excluait donc intentionnellement le troisième et le quatrième niveau de la nomenclature de l'INSEE, afin d'effectuer une comparaison entre deux groupes quantitativement homogènes [un peu moins de 800 personnes dans les deux groupes], mais qualitativement très différents du point de vue du caractère statistique retenu (les premiers ne possédaient pas le baccalauréat tandis que les seconds possédaient au moins un bac plus trois). L'intention de cette enquête était de voir si le niveau d'études pouvait limiter l'influence des biais cognitifs sur nos raisonnements ${ }^{8}$. Parmi les six problèmes posés, il y avait le problème des cartes introduit aussi dans les focus groups.

La réponse rouge-rouge [qui est la bonne réponse] n’est obtenue que par $42,25 \%$ des enquêtés 9

La question que nous nous posions était donc de savoir si le pourcentage d'individus se trompant pouvait diminuer grâce à une délibération préalable. La réponse apportée par les résultats des focus groups est: non. Le pourcentage d'erreur dans ce cas atteint $76 \%$. Pour obtenir ce calcul nous avons tenu compte des valeurs du consensus lorsqu'il s'en dégageait un et de la délibération obtenue par vote majoritaire lorsqu'il n'y en avait pas.

Dans le cas très spécifique des problèmes posés, il semble qu'il n’y ait pas de plus-value observable de la délibération quant à la performance des groupes. Le graphique suivant rappelle nos résultats.

7 Je tiens à remercier ici la promotion 2002-2003 de la licence pluridisciplinaire de l'université Nancy 2 sans l'aide matérielle de laquelle cette enquête n'aurait pas pu être réalisée ainsi que Francine Mansuy qui prit en charge la saisie informatique.

8 Pour les résultats de cette enquête, voir Bronner, 2008.

9 Mais alors que les diplômés sont à 52,2\% dans l'erreur, les non-diplômés se trompent dans $63,7 \%$ des cas $[\mathrm{chi} 2=21,88, \mathrm{ddl}=2, \mathrm{I}-\mathrm{p}=99,99 \%]$. 
Tiré à part adressé à Gérald Bronner

Figure 2. L'impact de la délibération collective sur les réponses

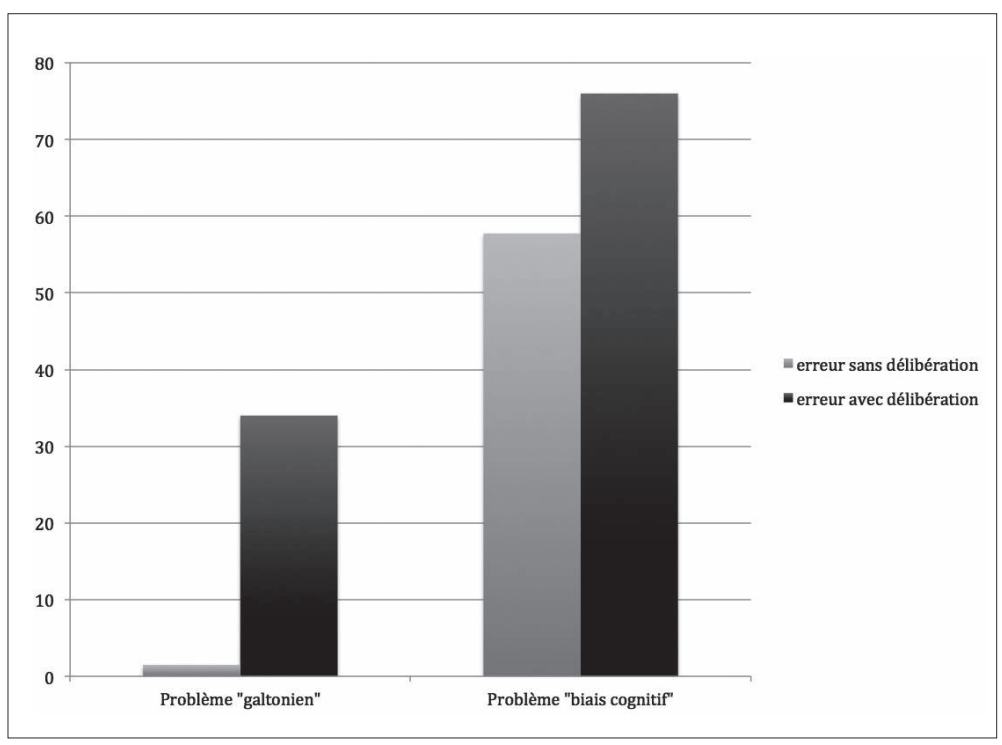

Conduire une enquête par focus groups, surtout lorsqu'on se donne des critères de sélection des volontaires très contraignant (dans notre cas : le fait qu’ils ne se connaissent pas préalablement), est lourd et complexe. Les résultats obtenus ici sont à prendre avec précaution car ils sont fondés sur des données quantitativement modestes. Celles-ci cependant vont au-delà des normes pratiquées dans de tels exercices notamment en psychologie cognitive. À ce titre, on doit souligner quelques rares autres tentatives dans la littérature pour répondre à la question de la performance des groupes pour résoudre des problèmes de biais cognitifs : Wright et Wells (1985) ; Argote, Seabright et Dyer (1986) ; Stasson, Kaoru, Zimmerman et Davis (1988). Cependant, les résultats obtenus restent franchement ambigus, ils montrent que, confrontés à divers biais cognitifs, les groupes peuvent suivre des processus de décision et de résolution de problèmes qui les conduisent, dans certains cas, à des performances inférieures à celles des individus, et, dans d'autres cas, à des performances supérieures. En aucun cas, cependant, ces biais ne disparaissent par la délibération. 
En plus de la prudence que l'on doit avoir concernant l'extrapolation de ces résultats, il ne s'agit pas non plus de conclure que la délibération est un élément nuisible à la décision collective. Il est facile de trouver des exemples qui contrediraient cette conclusion hâtive. En revanche, il est possible sur la base de ces résultats de mieux définir le périmètre des critères qui doivent présider aux réflexions sur la démocratie collaborative.

\section{CONCLUSION}

\section{Pour une ingénierie sociale de la sagesse des foules}

Ces réflexions sur la démocratie collaborative ont pour élément central, que l'on retrouve d'ailleurs dans la citation faite de Surowiecki plus haut, le critère de diversité. Ce critère a été imposé par Scott E. Page (2007) : «la diversité prime sur le compétence». On comprend parfaitement la pertinence de ce critère dans des structures de situations «galtoniennes » sans délibération puisqu'on suppose qu'elle doit pouvoir être corrélé à l'équirépartition de l'erreur. En revanche, exprimé inconditionnellement, il pose un double problème.

D’une part, la délibération elle-même peut être une entrave à l'expression de cette diversité car plutôt que produire une équiréparition des réponses elle peut, par des effets de cascade, de conformisme, d’autorité (celle-ci ne ressortant pas forcément de la qualité d'expert mais aussi de la qualité du capital argumentatif possédé par les uns ou les autres [Mendelberg, 2002]) ou d'ancrage. Pour l'écrire autrement, la délibération est aussi un phénomène social en soi qui peut dissuader certains de donner une réponse sincère à un problème posé, c'est particulièrement tangible dans les effets de «prisme d'équité» mentionnés plus haut.

D’autre part, toute la diversité sociale, politique, associative, etc., qui pourra caractériser un groupe, aussi large qu'on pourra la souhaiter, ne retirera rien au fait que chacun de ces individus est doté d'un cerveau qui, dans certaines circonstances, a des chances de fonctionner comme celui des autres. Il existe plusieurs lestes qui pèsent sur notre jugement et qui, parce qu'ils sont bien partagés, s'agrègent facilement dans des assemblées délibératives : biais d’incertitude (Tversky et Shafir, 1992) qui conduit à des suspensions inconséquentes du jugement, 
Tiré à part adressé à Gérald Bronner

surestimation des faibles probabilités (Prelec, 1998), balance inéquitable des coûts et des bénéfices (Tversky et Kahneman, 198I), difficulté de comprendre les effets de seuil, préférence pour l'examen des conséquences de nos actions plutôt que de nos inactions (Tversky et Kahneman, 1984), etc.

Ce point avait d'ailleurs été vu par Condorcet lui-même. Dans son célèbre Essai sur l'application de l'analyse à la probabilité des décisions rendues à la pluralité des voix qu'une assemblée (I785, p.clxxx) il formule un «théorème» stipulant que si dans une assemblée chaque votant a une probabilité de chance supérieure à $50 \%$ de prendre une bonne décision, alors plus l'assemblée sera importante, plus la probabilité qu'une décision collective, prise à la majorité, tende vers une conclusion optimale et rationnelle est grande. Ce théorème central de la démocratie délibérative est exprimé d'une autre façon par Hélène Landemore: «Dans la mesure, où la diversité cognitive est, jusqu’à un certain point, fonction du nombre de participants, la délibération au sein d'un groupe nombreux est, d’un point de vue épistémique, supérieure à la délibération au sein d'un petit groupe. » (Landemore, 20ı, p. I I). Deux ou trois personnes peuvent se tromper bien entendu, mais cette infortune, disparaît à l'horizon de l'improbabilité lorsque des groupes délibératifs de plus grande envergure se mettent en place. Pour éviter cette convergence de l'erreur, donc, la clause de diversité est fondamentale.

Condorcet avait pourtant lui-même vu que son théorème n'était que d’application restreinte et les commentateurs qui veulent croire en la sagesse des foules oublient souvent de mentionner ce point.

Nous savons à présent qu'un grand nombre d'individus n'implique pas mécaniquement la diversité cognitive pas plus que des jugements sages. Dans certaines circonstances, c'est même le contraire qui est vrai. Supposons qu'une erreur de raisonnement ait 60\% de chances de se manifester dans tout esprit. Celle-ci aura d'autant plus de chances d'être majoritaire dans une assemblée qui vote que le nombre de votants sera important. Au contraire, supposons que cette assemblée soit constituée d'une seule personne, alors il demeure 40\% de chances qu'elle prenne la bonne décision. C'est exactement ce que savait déjà 
Condorcet puisqu'il écrivait dans l'Essai sur l'application de l'analyse à la probabilité des décisions rendues à la pluralité des voix qu'une assemblée:

Ce que nous avons dit des inconvénients d'une assemblée trop nombreuse śapplique à plus forte raison au cas où la probabilité de la voix d'un certain nombre de Votants tombe au dessous de $1 / 2[\ldots]$. Lorsque la probabilité de la voix d'un votant tombe en-dessous de $\mathrm{I} / 2$, il doit y avoir une raison pour laquelle il prononce moins bien que ne le ferait le hasard; et cette raison ne peut être prise que dans les préjugés auxquels ce votant est soumis. (1785, p.clxxxij).

Dans ces conditions, le nombre de votant optimal est I.

La démocratie collaborative est un outil de prise de décision qui a de grandes vertus et qui est une ressource de renouvellement de la vie démocratique qui ne doit pas être négligée. Mais plutôt qu’une défense idéologique, elle a besoin d'une approche en termes d'ingénierie sociale qui précise ce à quoi on doit s'attendre face à telle structure de situation décisionnelle, dans tel contexte politique, informationnel, qui puisse répondre à la question de savoir quel nombre optimal de délibérants on doit intégrer pour être assurés que le temps de parole sera équitablement réparti, qui puisse réduire les effets pervers bien documentés par la psychologie sociale des échanges collectifs. Cette ingénierie sociale doit aussi tenir compte de l'existence d'invariants mentaux qui neutralisent le critère de diversité en intégrant aux formations qui précèdent classiquement ces exercices démocratiques (par exemple dans les sondages délibératifs de Fishkin ou dans l'expérience précoce menée par le député Jean-Yves Le Déaut [Boy, Donnet et Rouqueplo, 2000]) une attention à l'existence de ces biais cognitifs et, d'une façon générale à une forme de pensée analytique de nature à faire reculer l'attraction que constituent certains types de raisonnements fautifs (Tran, Furnham et al., 20I4 ; Gervais et Norenzayan, 20I2). Pour répondre à toutes ces questions et à quelques autres qui en ferait un outil de renouvellement de la vie démocratique, l'intelligence des foules doit devenir une question épistémique. 


\section{BIBLIOGRAPHIE}

ARGOTE L., SEABRIGHT M.A. et DYER L., 1986, «Individual versus Group use of Base-Rate and Individuating Information», Organisational Behavior and Human Decision Processes, 38, p. 65-75.

ASCH S., 1946, «Forming Impressions of Personality», Journal of Abnormal and Social Psychology, 4I, p. 258-290.

-, 1955, «Opinions and Social Pressure», Scientific American, 193, p. 31-35.

BARBER B., 1984, Strong Democracy: Participatory Politics for a New Age, Berkeley, University of California Press.

BLOMBERG B. et HERRINGTON J., 2000, «A Theory of Flexible Moderates and Rigid Extremists with an Application to the U.S. Congress $\gg$, American Economic Review, 90, p. 605-620.

BRONNER G., 2007, L'Empire de l'erreur. Éléments de sociologie cognitive, Paris. PUF.

-, 2008, «Les normes du raisonnement: entre inné et acquis», Philosophia Scientae, 9, p. 193-213.

-, 2013, La Démocratie des crédules, Paris, PUF.

-, 2014, «Ressources et impasses du travail collaboratif», in La Confiance en commun, Paris, Textuel, 2014, p. 30-39.

BOY D., DONNET K.D. et ROUQUEPLO P., 2000, «Un exemple de démocratie participative: la "conférence de citoyens" sur les organismes génétiquement modifiés», 50-4, p.779-8I0.

CALLON M., LASCOUMES P. et BARTHE Y., 200I, Agir dans un monde incertain, Paris, Éditions du Seuil.

CARDON D., 2010, La Démocratie Internet, Paris, Éditions du Seuil.

ELSTER J. 1988, «Deliberation and Constitution Making», in J. Elster (éd.) Deliberative Democracy, Cambridge, Cambridge University Press, p. 97-122.

FISHKIN J.S., 2009, When the People Speak. Deliberative Democracy and Public Consultation, Oxford, Oxford University Press.

GERVAIS W. et NORENZAYAN A., 2012, «Analytic Thinking Promotes Religious Disbelief», Science, 336-6080, p.493-496. 
Revue européenne des sciences sociales

Tiré à part adressé à Gérald Bronner

GIRARD C. et LEGOFF A. 2010, La Démocratie délibérative. Anthologie de textes fondamentaux, Paris, Hermann.

GORDON K.H., 1924, «Group Judgments in the Field of Lifted Weights», Journal of Experimental Psychology, 7, p. 437-443.

KAHNEMAN D, 2012, Système I Système 2. Les deux vitesses de la pensée, Paris, Flammarion.

KRAUSE J., RUXTON G.D. et KRAUSE S., 2010, «Swarm Intelligence in Animals and Humans», Trends in Ecology and Evolution, 25-I, p. 28-34.

LANDEMORE H., 20I0, «La raison démocratique: les mécanismes de l'intelligence collective en politique», in J. Elster et H. Landmore (éds), La Sagesse collective. Raison Publique, 12, p. 9-55.

LEADBEATER C. et MILLER P., 2004, The Pro-Am Revolution: How Enthusiasts Are Changing Our Economy and Society, Londres, Demos.

LE BON G., 2003 (I895), La Psychologie des foules, Paris, PUF.

LOVELUCK B., 2008, «Internet, vers la démocratie radicale?», Le Débat, 4-|5|, p. $150-166$.

MACKAY C., 1995 (1845), Extraordinary Popular Dilusion and the Madness of Crowds, London, Wordwoth Editions.

MAHMOODI A. et al., 2015, «Equality bias impairs collective decision-making across cultures $\gg$, Proceedings of the National Academy of Sciences: <www.pnas.org/cgi/doi// 0.1073/pnas.1421692112>.

MENDELBERG T., 2002, «The Deliberative Citizen. Theory and Evidence», Resarch in Micropolitics, 6, p. 151-192.

MOREL C., 2012, Les Décisions absurdes 2, Paris, Gallimard.

PAGE S., 2006, The Difference. How the Power of Diversity Creates Better Groups. Firms, Schools, and Societies, Princeton, Princeton University Press.

PATEMAN C., 1970, Participation and Democratic Theory, Cambridge England, Cambridge University Press.

PLATON, 2002, La République, Paris, Garnier-Flammarion. 
Tiré à part adressé à Gérald Bronner

PRELEC D., 1998, «The probability weighting function», Econometrica, 47, p. 313-327.

ROSANVALLON P. 2006, La Contre-démocratie. La politique à l'âge de la défiance, Paris, Éditions du Seuil.

SHERIF M., 1937, «An Experimental Approach to the Study of Attitudes», Sociometry, I, p.90-98.

STASSON M.F., KAORU O., ZIMMERMAN S.K. et DAVIS J.H., 1988, «Group Consensus Processes on Cognitive Bias Tasks: A Social Decision Scheme Approach», Japanese Journal of Psychology, 30, p. 68-77.

SUNSTEIN C.R., 2003, Why Societies Need Dissent?, Cambridge, Harvard University Press.

-, 2006, Infotopia: How Many Minds Produce Knowledge, Londres et Oxford, Oxford University Press.

SUROWIECKI J., 2008 (2004), La Sagesse des foules, Paris, Lattès.

TRAN U.S., FURNHAM A. et al., 2014, «Analytic Thinking Reduces Belief in Conspiracy Theories», Cognition, 133, p. 572-585.

TVERSKY A. et KAHNEMAN D., 198I, Science, 2II-448I, p.453-458.

-, 1984, «The Simulation Heuristic», in TVERSKY, KAHNEMAN et SLOVIC, 1984, p. 160-173.

- et SLOVIC P. (éds), 1984, Judgment Under Uncertainty: Heuristics and Biaises, Cambridge, Cambridge University Press.

TVERSKY A. et SHAFIR E., 1992, «The Disjunction Effect in Choice under Uncertainty», Psychological Science, 3-5, p. 305-309.

WRIGHT E. F. et WELLS G. L., 1985, «Does Group Discussion Attenuate the Dispositional Bias?», Journal of Applied Social Psychology, I5, p. 531-546. 